\title{
NeuroImage
}

\section{Less white matter concentration in autism: 2D voxel-based morphometry}

\author{
Moo K. Chung, ${ }^{\mathrm{a}, \mathrm{b}, \mathrm{c}, *}$ Kim M. Dalton, ${ }^{\mathrm{c}}$ Andrew L. Alexander, ${ }^{\mathrm{c}, \mathrm{d}, \mathrm{e}}$ and Richard J. Davidson ${ }^{\mathrm{c}, \mathrm{e}, \mathrm{f}}$ \\ ${ }^{a}$ Department of Statistics, University of Wisconsin-Madison, Madison, WI 53706, USA \\ ${ }^{\mathrm{b}}$ Department of Biostatistics and Medical Informatics, University of Wisconsin-Madison, Madison, WI 53706, USA \\ ${ }^{\mathrm{c}}$ Keck Laboratory for Functional Brain Imaging and Behavior, University of Wisconsin-Madison, Madison, WI 53706, USA \\ ${ }^{\mathrm{d}}$ Department of Medical Physics, University of Wisconsin-Madison, Madison, WI 53706, USA \\ ${ }^{\mathrm{e}}$ Department of Psychiatry, University of Wisconsin-Madison, Madison, WI 53706, USA \\ ${ }_{\mathrm{f}}^{\mathrm{f}}$ Department of Psychology, University of Wisconsin-Madison, Madison, WI 53706, USA
}

Received 1 February 2004; revised 28 March 2004; accepted 21 April 2004

\begin{abstract}
Autism is a neurodevelopmental disorder affecting behavioral and social cognition, but there is little understanding about the link between the functional deficit and its underlying neuroanatomy. We applied a 2D version of voxel-based morphometry (VBM) in differentiating the white matter concentration of the corpus callosum for the group of 16 high functioning autistic and 12 normal subjects. Using the white matter density as an index for neural connectivity, autism is shown to exhibit less white matter concentration in the region of the genu, rostrum, and splenium removing the effect of age based on the general linear model (GLM) framework. Further, it is shown that the less white matter concentration in the corpus callosum in autism is due to hypoplasia rather than atrophy.
\end{abstract}

(C) 2004 Elsevier Inc. All rights reserved.

Keywords: Autism; Voxel; Morphometry

\section{Introduction}

Autism is a neurodevelopmental disorder of brain function that has begun to attract in vivo structural magnetic resonance imaging (MRI) studies in the region of the corpus callosum (Egaas et al., 1995, Hardan et al., 2000; Manes et al., 1999; Piven et al., 1996, 1997). There is little understanding about the link between the functional deficit and the underlying abnormal anatomy in autism, which provides motivation for our study. These studies use the Witelson partition or a similar partition scheme of the corpus callosum (Witelson, 1989). Witelson partitioned the midsagittal cross-sectional images of the corpus callosum along the maximum anterior-posterior line (Talairach and Tournoux, 1988) and defined the region of the genu, rostrum, midbodies, isthmus, and splenium from the anterior to posterior direction. Based on the Witelson

* Corresponding author. Department of Statistics, University of Wisconsin-Madison, 1220 West Dayton Street, Madison, WI 53706. Fax: +1-608-262-0032.

E-mail address: mchung@stat.wisc.edu (M.K. Chung).

URL: http://www.stat.wisc.edu/_mchung.

Available online on ScienceDirect (www.sciencedirect.com.) partition, there has been a consistent finding in abnormal reduction in anterior, midbody, and posterior of the corpus callosum (Brambilla et al., 2003a,b).

Piven et al. (1997) compared 35 autistic individuals with 36 normal control subjects controlling for total brain volume, gender, and IQ and detected a statistically significant smaller midbody and posterior regions of the corpus callosum in the autistic group. Manes et al. (1999) compared 27 low functioning autistic individuals with 17 normal controls adjusting for the total brain volume. They found a smaller corpus callosum compared to the control group in genu, rostrum, anterior midbody, posterior midbody, and isthmus but did not find statistically significant differences in the rostrum and the splenium, although the sample mean of the rostrum and splenium size are smaller than that of the control group. Hardan et al. (2000) compared 22 high functioning autistic to 22 individually matched control subjects and showed smaller genu and rostrum of the corpus callosum adjusting for the total brain volume based on the Witelson partition. The smaller corpus callosum size was considered as an indication of a decrease in interhemispheric connectivity. They did not detect other regions of significant size difference. For an extensive review of structural MRI studies for autism that have been published between 1966 and 2003, one may refer to Brambilla et al. (2003a,b).

The shortcoming of the Witelson partition is the artificial partitioning. The Witelson partition may dilute the power of detection if the anatomical difference occurs near the partition boundary. Alternative voxel-wise approaches that avoid predefined regions of interests (ROI) have begun to be used in structural autism studies. Vidal et al. (2003) used the tensor-based morphometry (TBM) to show reduced callosal thickness in the genu, midbody, and splenium in autistic children. Hoffmann et al. (2004) used a similar TBM to show curvature difference in the midbody. Abell et al. (1999) used voxel-based morphometry (VBM) (Ashburner and Friston, 2000, 2001; Good et al., 2001a,b; Wright et al., 1995) in high functioning autism to show decreased gray matter volume in the right paracingulate sulcus, the left occipito-temporal cortex, and increased amygdala and periamygdaloid cortex.

The advantage of the VBM framework over the Witelson partition approach is that it is completely automated and does 
not require artificial partitioning of the corpus callosum that introduces undesirable bias. Further, it is not restricted to a priori ROIs enabling us to perform the statistical analysis at each voxel level and to pinpoint the exact location of the anatomical differences within ROI even if there is no ROI size differences. Although VBM was originally developed for whole-brain 3D morphometry, our study concentrates on the midsagittal crosssectional corpus callosum regions to be able to compare the result with the previous 2D Witelson partition studies such as Hardan et al. (2000), Manes et al. (1999), and Piven et al. (1997). Hence, we will prefer 2D VBM over 3D VBM in this study. Let us review the basis of VBM and its connection to ROI morphometry briefly.

\section{Methods}

Voxel-based morphometry

VBM as implemented in SPM'99 computer package (Wellcome Department of Cognitive Neurology, London, UK, http://www. fil.ion.ucl.ac.uk/spm) starts with normalizing each structural MRI to the standard SPM template and segmenting it into white and gray matter and cerebrospinal fluid based on a Gaussian mixture model (Ashburner and Friston, 1997, 2000). Based on a prior probability of each voxel being the specific tissue type, a Bayesian approach is used to get a better estimate of the posterior probability. This Bayesian update of the probability is iterated many times until the probability converges. This probability is usually called density. Note that this is not physical density so it should be interpreted probabilistically. There has been on going discussions on the amount of image registration and modulation by the determinant of the Jacobian of deformation fields in VBM (Ashburner and Friston, 2001; Bookstein, 2001; Mehta et al., 2003).

In our study, we applied the 2D version of VBM on the midsagittal cross section of 3D MRI (Fig. 1). Let $p(x) ; x \in \mathbb{R}^{2}$ be the white matter density of the $2 \mathrm{D}$ midsagittal cross section of corpus callosum $\Omega$. Denote $1_{\Omega}(x)=1$ if $x \in \Omega$ and 0 otherwise. The shape of $\Omega$ is random and we associate it with probability $p(x)$,

$P(x \in \Omega)=p(x)$.

Then the area of the corpus callosum $\Omega$ is given by

$A(\Omega)=\int 1_{\Omega}(x) \mathrm{d} x$.

Since we are dealing with the normalized corpus callosum after image registration in SPM'99, the area is the relative measure. The absolute area can be estimated by multiplying the determinant of the Jacobian of the deformation (Good et al., 2001a,b; Shen and Davatzikos, 2003). However, we are not interested in detecting the global corpus callosum size between groups but rather a local shape difference after registration. Since we need to do a statistical analysis after controlling for the total corpus callosum size, it is not necessary to estimate the absolute area and we will perform the statistical analysis controlling for the relative area.

From Eq. (1), we estimate the area of corpus callosum as

$\hat{A}(\Omega)=\mathbb{E} A(\Omega)=\int \mathbb{E} 1_{\Omega}(x) \mathrm{d} x=\int p(x) \mathrm{d} x$.

So the sum of the white matter density over all voxels gives an approximation to the area of the corpus callosum. In this context, ROI morphometry used in Piven et al. (1997), Hardan et al. (2000), and Manes et al. (1999) can be viewed as a subset of VBM framework. For instance, ROI morphometry based on Witelson partition can be performed by segmenting the normalized $2 \mathrm{D}$ midsagittal images.

The white matter density is a probability ranging between 0 and 1 so it is not exactly normally distributed. To make it more normal, one may apply the logit transform (Ashburner and Friston, 2000). However, we did not perform the logit transform since we adapted the usual Gaussian kernel smoothing with relatively large 15-pixel-wide FWHM to make the white matter density more normal (Fig. 1). For detailed distributional assumptions in VBM, one may refer to Ashburner and Friston (2000) and Salmond et al. (2002). Let $K_{\sigma}$ be a Gaussian kernel that follows bivariate normal $N\left(0 ; \sigma^{2} I\right)$. Kernel smoothing of scalar function $p$ is defined as convolution

$\int K_{\sigma}^{*} p(x) \mathrm{d} x=\iint K_{\sigma}(x-y) p(y) \mathrm{d} y \mathrm{~d} x=\int p(y) \mathrm{d} y$.

From Eqs. (2) and (3), it can be seen that the area estimate

$\hat{A}(\Omega)=\int K_{\sigma}^{*} p(x) \mathrm{d} x$

is invariant under scale $\sigma$ change. This can be used to estimate the corpus callosum area from VBM white matter density maps. Assuming normality for kernel smoothing $K_{\sigma} * p, \hat{A}(\Omega)$ would be normal so the usual statistical tests based on normality can be applicable.

We have numerically checked the result (Eq. (4)) comparing the area estimates with different scales $\sigma=\delta$ FWHM and $\sigma=10 \delta$

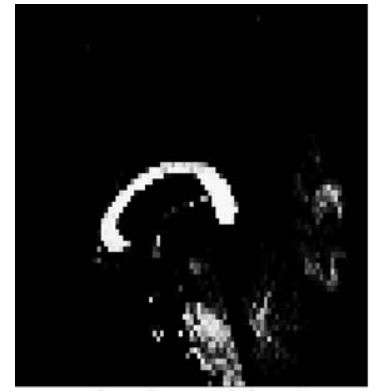

single subject

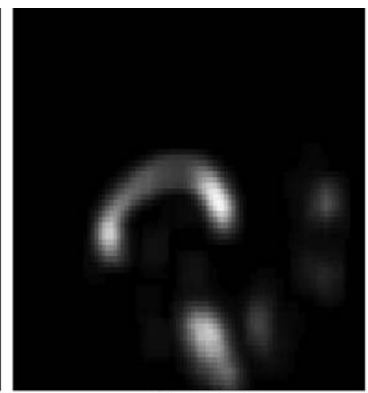

smoothed image

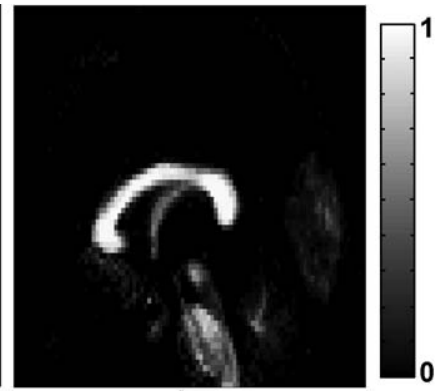

sample mean

Fig. 1. Left: white matter segmentation of an individual midsagittal MRI from SPM'99. Middle: 2D Gaussian kernel smoothing of the white matter segmentation with 12 pixel wide FWHM. Right: the sample mean of the smoothed white matter density for control groups. 
Table 1

Age and relative total corpus callosum area distribution

\begin{tabular}{|c|c|c|c|c|c|c|c|c|c|c|c|c|c|c|c|c|}
\hline \multicolumn{17}{|c|}{ Control } \\
\hline Age & 15 & 18 & 18 & 16 & 15 & 13 & 18 & 15 & 21 & 17 & 16 & 23 & & & & \\
\hline Area & 154 & 237 & 225 & 216 & 216 & 246 & 156 & 195 & 175 & 223 & 236 & 194 & & & & \\
\hline \multicolumn{17}{|c|}{ Autistic } \\
\hline Age & 15 & 20 & 17 & 13 & 12 & 15 & 25 & 14 & 15 & 14 & 24 & 18 & 10 & 12 & 22 & 12 \\
\hline Area & 146 & 238 & 183 & 161 & 104 & 214 & 208 & 238 & 231 & 183 & 198 & 207 & 206 & 174 & 193 & 183 \\
\hline
\end{tabular}

FWHM (FWHM is the full width at the half maximum and $\delta=2$ $\mathrm{mm}$ is the resolution of image). The corpus callosum area estimate does not differ by more than $0.6 \%$ in average when scales differ by 10 times. The numerical difference is due to the truncation of the Gaussian kernel. The total area estimates are given in Table 1. Again it should be pointed out that these are relative area estimates after normalization.

\section{Two sample t test}

Let $p_{a}^{1}, \cdots, p_{a}^{m}(m=16)$ be the white matter density for autism and $p_{c}^{1}, \cdots, p_{c}^{n}(n=12)$ be the white matter density for controls at a given voxel. We assume $p_{a}^{i} \sim N\left(\mu_{a}, \sigma_{a}^{2}\right)$ and $p_{c}^{j} \sim N\left(\mu_{c}, \sigma_{c}^{2}\right)$ independently. We denote the sample mean and the variance of $p_{a}^{i}$ and $p_{c}^{j}$ by $\bar{p}_{a}, \bar{p}_{c}, S_{a}^{2}, S_{c}^{2}$, respectively. First we test if the autistic group has more white matter variability in the corpus callosum:

$H_{0}: \sigma_{a}^{2}=\sigma_{c}^{2}$ vs. $H_{1}: \sigma_{a}^{2} \geq \sigma_{c}^{2}$.

Under the null assumption, the ratio of the sample variances $S_{a}^{2}=S_{c}^{2}$ has an $F$ distribution with $m-1$ and $n-1$ degrees of freedom. The $F$ map is given in Fig. 2 where the splenium of the autistic group shows statistically significant larger variability. In this region, the autism shows nine times more variability ( $P$ value of 0.0004$)$. Now we test if the white matter density difference between the autistic group and the control group is statistically significant (Fig. 3):

$H_{0}: \mu_{a}=\mu_{c}$ vs. $H_{1}: \mu_{a} \leq \mu_{c}$,

The classical test statistic $T$ for the Behrens-Fisher problem depends on the unknown nuisance parameter $\sigma_{a}$ and $\sigma_{c}$ that are estimated by sample variances (Tsui and Weerahandi, 1989):

$$
T=\frac{\bar{d}_{a}-\bar{d}_{c}}{\sqrt{S_{a}^{2} / m+S_{c}^{2} / n}} \sim t_{d f}
$$

where the degrees of freedom $d f$ of the $t$ distribution is estimated by

$$
d f=\frac{\left(S_{a}^{2} / m+S_{c}^{2} / n\right)^{2}}{\left(S_{a}^{2} / m\right)^{2} /(m-1)+\left(S_{c}^{2} / n\right)^{2} /(n-1)} .
$$

However, since the degrees of freedom $d f=m+n-2=26$ is relatively high in our study, we may pool the variance and use the pooled two sample $t$ statistic

$$
T=\frac{\bar{d}_{a}-\bar{d}_{c}}{S_{p} \sqrt{1 / m+1 / n}} \sim t_{m+n-2},
$$

where the pooled variance $S_{p}^{2}=\left((m-1) S_{a}^{2}+(n-1) S_{c}^{2}\right) /(m+n-$ $2)$. In fact, we did not see much difference between $t$ statistic images in two cases so we will pool the variances for the two sample $t$ test. The advantage of pooling the variance is that the statistical distribution becomes exact. However, care should have taken when pooling the variance since it can be shown to lead to erroneous conclusions when it is applied when population variance are different for small sample size. An alternate approach would be to use the concept of generalized $P$ value, which provides exact probability statement in the presence of nuisance parameters (Tsui and Weerahandi, 1989).

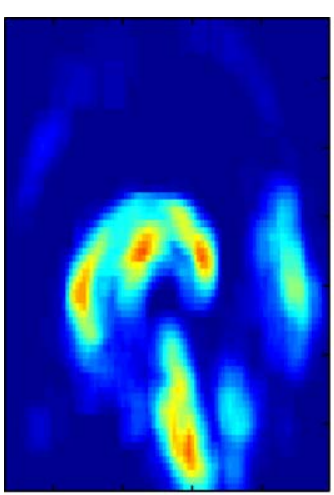

autism

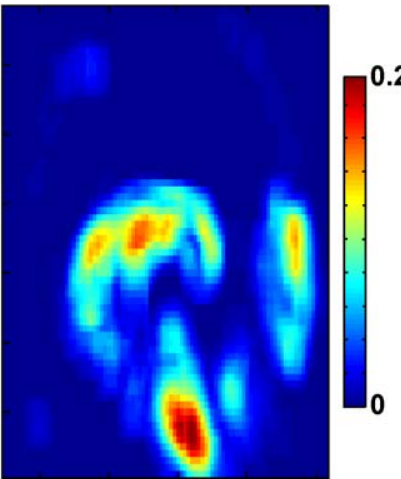

control

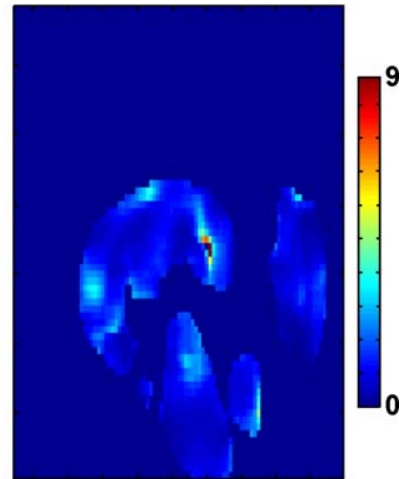

F map

Fig. 2. The sample standard deviations of autism (left) and control (middle), respectively. The last figure shows the $F$ map showing up to nine times more variability of the white matter concentration in the splenium of the autistic group. The genu and the midbody also show larger white matter concentration variability but they are not as predominant as the splenium. 


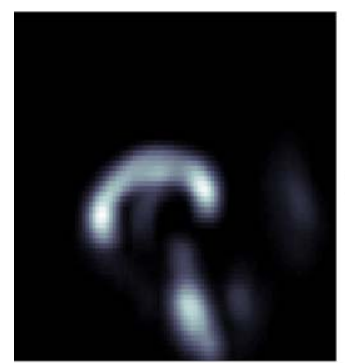

autism

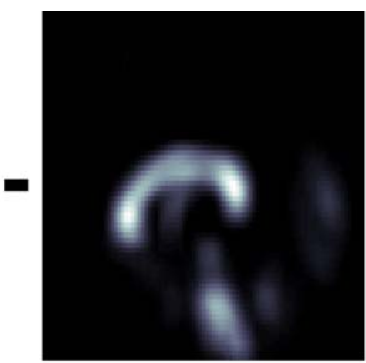

control

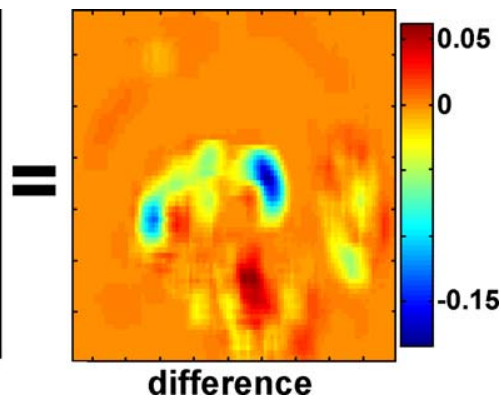

difference

Fig. 3. The sample mean of the white matter density of the autistic group (left) and the control group (middle), respectively. The third figure is the difference of the sample mean. We are interested in testing if this difference is statistically significant. Simply comparing the white matter density difference, the autistic group shows less white matter concentration in the genu and the splenium of the corpus callosum.

In the place of a test statistic, they use so-called generalized test variable and the corresponding $P$ value can be computed exactly.

\section{General linear models}

Since all subjects are different in age, brain size, and IQ, there might be confounding effects of age, brain size, and IQ on the white matter density. Previous anatomical studies in the corpus callosum suggest this (Brambilla et al., 2003a,b). On the other hand, deformation-based morphometry and tensor-based morphometry in the normal developmental studies in children show that there is relative brain tissue growth in the corpus callosum over time (Chung et al., 2001; Thompson et al., 2000). In particular, Chung et al. (2001) showed white matter local volume increase in the midbody, isthmus, and splenium of the corpus callosum in 28 normal subjects from 12 to 16 years. In our study, the age for the control group is $17.1 \pm 2.8$, and for the autism group, it is $16.1 \pm$ 4.5 years (Table 1). The age ranges for two groups are somewhat compatible; however, there might be still age effect on the white matter difference. To evaluate any possible effect of age on the white matter density, we first fit linear model

density $=\lambda_{1}+\lambda_{2} \cdot$ age

to each group separately using the least-squares method at each voxel (Figs. 4 and 5). The liner model fits show the dynamic pattern of different white matter density changes over time between groups. The pattern of growth in the corpus callosum is different. The autistic group shows lower white matter density compared to the control group at the lower age, but gains white matter over time while the control group shows decreasing white matter density with age. There thus appears to be age differences for at least some regions of the corpus callosum and these should be accounted for.

One approach for removing the age effect would be to modulate the white matter density such that the age effect will not be present. First, we estimate $\lambda_{1}$ and $\lambda_{2}$ for each group via the least-squares method. Then, adjust the white matter density $d(t)$ at time $t$ via transform

$d(t) \rightarrow d(t)+\hat{\lambda}_{2}(\bar{t}-t)$

where $\hat{\lambda}_{2}$ is the least-squares estimation of $\lambda_{2}$ and $\bar{t}$ is the mean age of both the controls and autistic combined together. This has an effect of modulating the densities measured at different age to fixed reference age $\bar{t}$. A more general approach would be to use a general linear model (GLM). The general linear model (GLM) is a flexible framework that can be used in localizing the region of white matter concentration that are related to covariates such as age, IQ, brain size, gender, and handedness. We consider the following GLM:

density $=\lambda_{1}+\lambda_{2} \cdot$ age $+\beta_{1} \cdot$ group $+\epsilon$,

where the dummy variable group is 1 for autism and 0 for control. To control the possible effect of the corpus callosum size difference, we will also consider following GLM separately:

density $=\lambda_{1}+\lambda_{2} \cdot$ age $+\lambda_{3} \cdot$ area $+\beta_{1} \cdot$ group $+\epsilon$,

where area is the relative total corpus callosum area given in Eq. (2). In these formulations, we do not have separate linear equations as before but combine autism and control group data together and have a single linear equation. A similar linear model formulation in the VBM is used in localizing the region of the gray matter maturation in children (Paus et al., 1999). To formulate the problem in somewhat general fashion, let $z=\left(z_{1}\right.$, $\left.\cdots, z_{k}\right)$ to be nuisance variables such as age and area and $x=\left(x_{1}\right.$, $\left.\cdots, x_{p}\right)$ to be the variable of interest such as group. Then we have GLM in the following form

$p=z \lambda+x \beta+\epsilon$

where $\lambda=\left(\lambda_{1}, \cdots, \lambda_{k}\right)^{\prime}$ and $\beta=\left(\beta_{1}, \cdots, \beta_{p}\right)^{\prime}$. We assume the usual zero mean Gaussian noise. Then we test if the group is significant, that is,

$H_{0}: \beta=0$ vs. $H_{1}: \beta \neq 0$

The fit of model is measured by the residual sum of squares or the sum of the squared errors (SSE) that are given in Chung et al. (2004). Then under $H_{0}$,

$F=\frac{\left(\mathrm{SSE}_{0}-\mathrm{SSE}_{1}\right) / p}{\mathrm{SSE}_{0} /(m+n-p-k)} \sim F_{p, m+n-p-k}$.

The larger the $F$ value, it is more unlikely to accept $H_{0}$. For testing group difference controlling for age, $k=2$ and $p=1$, while 

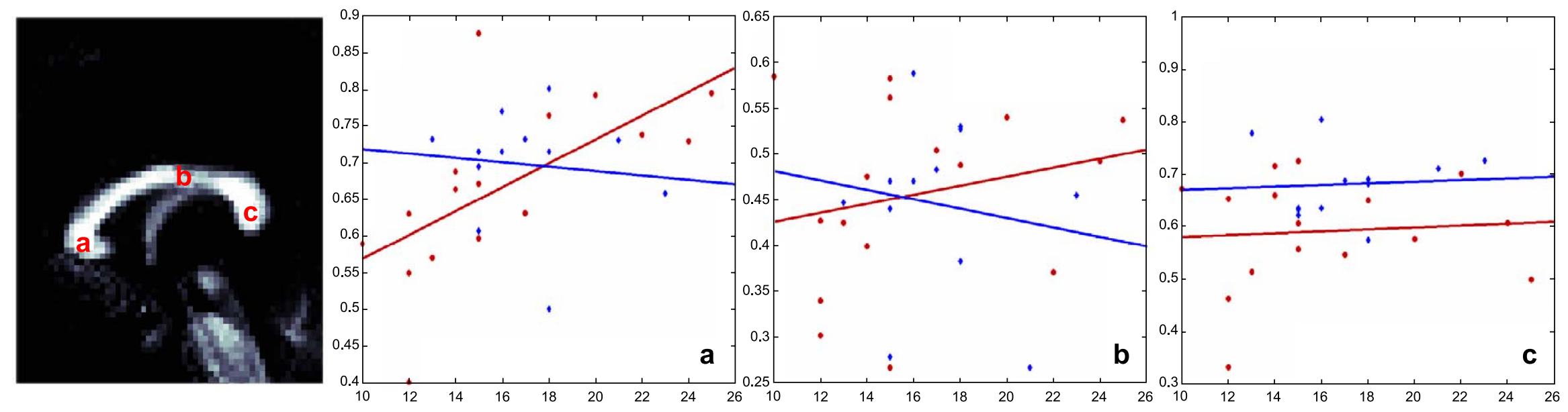

Fig. 4. The simple linear fit correlating the white matter density to age at genu (a), midbody (b), and splenium (c). One specific pixel is chosen at each region to illustrate the different dynamic pattern of the density change. Red color is the autism group and blue color is the control group. The splenium seems to show obvious white matter deficiency in autism but group differences in the genu and the midbody are unclear due to different associations with age in each group. 


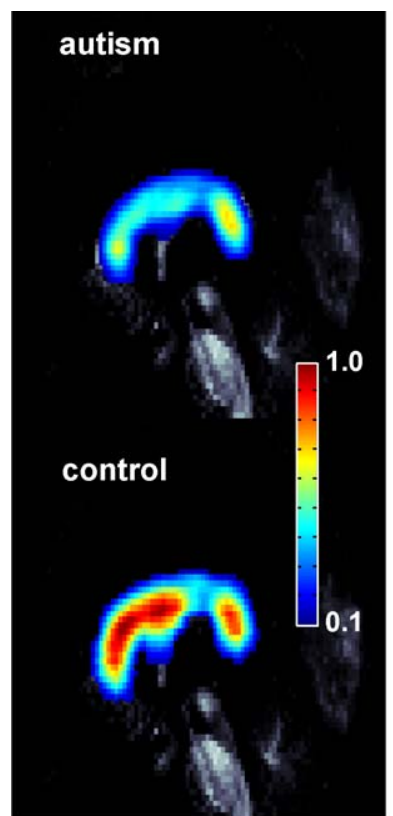

intercept

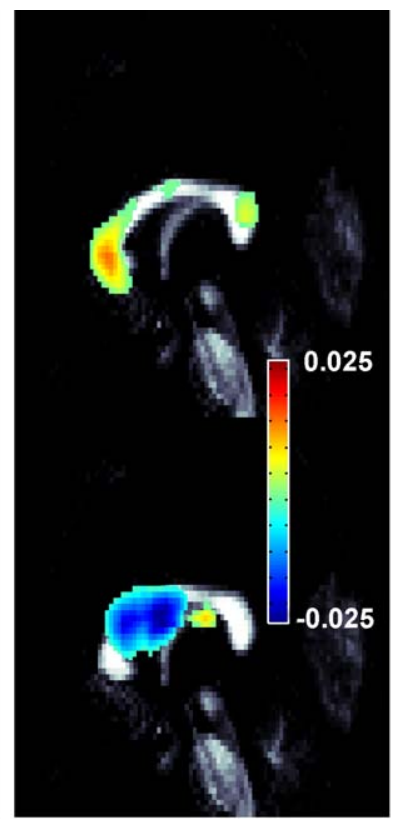

slope

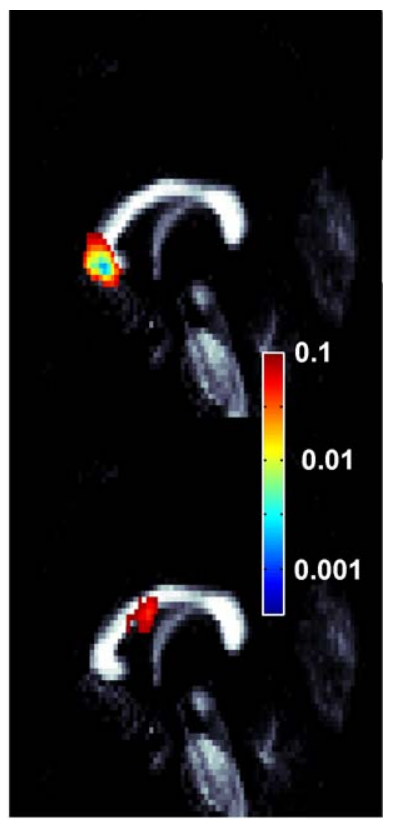

p-value

Fig. 5. Linear regression of the white matter density on age. Linear growth model density $=\lambda_{1}+\lambda_{2} \cdot$ age is fitted for each group separately. $\lambda_{1}$ is the intercept and $\lambda_{2}$ is the slope of the regression line. The autistic group shows relatively lower white matter density compared to the control at lower age but gains white matter over time while the control group shows decreasing white matter density with age. The control group shows $2.5 \%$ per year decrease of white matter in the midbody while the autistic group shows $2.5 \%$ per year increase of white matter in the genu. White matter decrease in the midbody of the control subjects is not significant (uncorrected $P$ value, 0.1 ; corrected $P$ value, close to 1 ). $F$ test was performed for the fit of the linear model and its $P$ value is computed.

for testing group difference controlling for both age and area, $k=3$ and $p=1$.

For multiple comparisons that account for spatially correlated error, one may use the result of the random field theory (Worsley, 1994; Worsley et al., 1996), the false discovery rates (Benjamini and Hochberg, 1995; Genovese et al., 2002), or permutation tests (Nichols and Holmes, 2002). If $F(x)$ is a smooth $F$ field (Worsley, 1994), the corrected $P$ value for correcting multiple comparisons over the all pixels in the corpus callosum $\Omega$ is given by

$P\left(\sup _{x \in \Omega} Y(x)>h\right) \approx \sum_{d=0}^{2} \mu_{d}(\Omega) \rho_{d}(h)$

where $\mu_{d}$ are the $d$ dimensional Minkowski functionals and $\rho_{d}$ are the $d$ dimentional Euler characteristic (EC) density (Worsley et al., 1996). For nonregular jagged shapes such as the corpus callosum, the Minkowski functionals can be estimated in the following fashion. Treating pixels inside $\Omega$ as points on a lattice, $V$ is the number of subpoints, $E$ is the number of edges connecting each adjacent lattice points, $F$ is the number of faces formed by four connected edges. Then, $\mu_{0}=\chi(\Omega)=V-E+F=1, \mu_{1}=(E-2 F) \delta$, $2 F) \delta, \mu_{2}=F \delta^{2}$ where $\delta=2 \mathrm{~mm}$ is the resolution of the image (Worsley et al., 1995). To find the number of edges and pixels contained in $\Omega$, we start from an initial face $F_{0}$ in the splenium of $\mathrm{CC}$ and add an additional face in the lattice one by one while counting the additional edges and faces. Our data $\Omega$ is taken to be the region of the white matter density bigger than 0.2 and we found $E=857$ and $F=392$. Thus, $\mu_{0}=1, \mu_{1}=73 \delta, \mu_{2}=392 \delta^{2}$ (Fig. 6). A compatible approach for computing Minkowski functionals for jagged irregular shapes has been implemented in
FMRISTAT package (http://www.math.mcgill.ca/keith/fmristat). The EC-density for $F$ field with $\alpha$ and $\beta$ degrees of freedom is given by

$$
\begin{aligned}
& \rho_{0}= \int_{h}^{\infty} \frac{\Gamma\left(\frac{\alpha+\beta}{2}\right)}{\Gamma\left(\frac{\alpha}{2}\right) \Gamma\left(\frac{\beta}{2}\right)} \frac{\alpha}{\beta}\left(\frac{\alpha x}{\beta}\right)^{\frac{(\alpha-2)}{2}}\left(1+\frac{\alpha x}{\beta}\right)^{-\frac{(\alpha+\beta)}{2}} \mathrm{~d} x, \\
& \rho_{1}=\lambda^{1 / 2} \frac{\Gamma\left(\frac{\alpha+\beta-1}{2}\right) 2^{\frac{1}{2}}}{\Gamma\left(\frac{\alpha}{2}\right) \Gamma\left(\frac{\beta}{2}\right)}\left(\frac{\alpha h}{\beta}\right)^{\frac{(\alpha-1)}{2}}\left(1+\frac{\alpha h}{\beta}\right)^{-\frac{(\alpha+\beta-2)}{2}}, \\
& \rho_{2}=\lambda \frac{\Gamma\left(\frac{\alpha+\beta-2}{2}\right)}{\Gamma\left(\frac{\alpha}{2}\right) \Gamma\left(\frac{\beta}{2}\right)}\left(\frac{\alpha h}{\beta}\right)^{\frac{(\alpha-2)}{2}}\left(1+\frac{\alpha h}{\beta}\right)^{-\frac{(\alpha+\beta-2)}{2}} \\
& \quad \times\left[(\beta-1) \frac{\alpha h}{\beta}-(\alpha-1)\right],
\end{aligned}
$$

where $\lambda$ measures the smoothness of fields and it is given by $\lambda=4 \ln 2 /\left(2 \pi \delta^{2}\right.$ FWHM $)$.

\section{Subjects and image acquisition}

Gender and handedness affect the corpus callosum anatomy (Luders et al., 2003; Pettey and Gee, 2002; Witelson, 1985, 1989) so all the 16 autistic and 12 control subjects used in this study are 


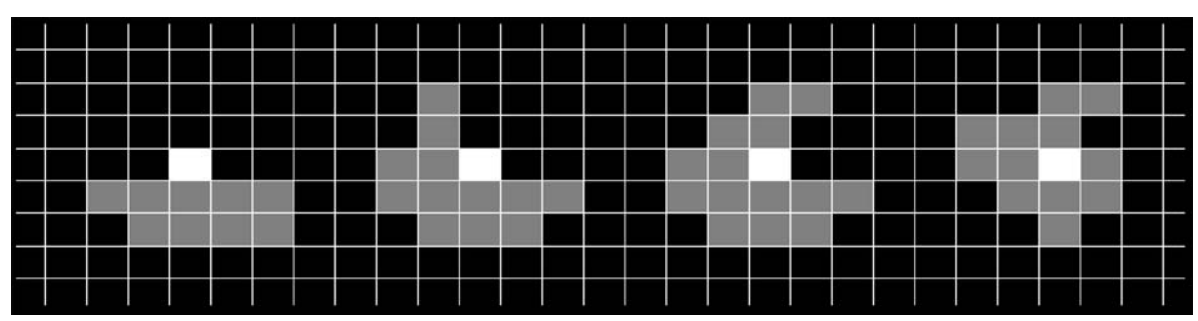

Fig. 6. There are only four possible configuration of adding additional face (white) to already searched regions (gray). Each of the above case corresponds to adding $3,2,1$, and 0 edges.

right-handed males except one subject who is ambidextrous. Sixteen autistic subjects were recruited for this study from a list of individuals with a diagnosis of high functioning autism in the Madison and Milwaukee area maintained for research purposes by the Waisman center at the University of Wisconsin-Madison. Diagnoses were confirmed with the Autism Diagnostic InterviewRevised (ADI-R) or clinical interview administered by a trained and certified psychologist at the Waisman center. All participants met DSM-IV criteria for autism or Asperger's pervasive developmental disorder. Twelve healthy, typically developing males with no current or past psychological diagnoses served as a control group. The average age for the control subject is $17.1 \pm 2.8$ and the autistic subjects is $16.1 \pm 4.5$, which is in compatible age range. The age and relative total area distribution for each group are given in Table 1.

High-resolution anatomical MRI scans were obtained using a 3Tesla GE SIGNA (General Electric Medical Systems, Waukesha, WI) scanner with a quadrature head RF coil. A three-dimensional, spoiled gradient-echo (SPGR) pulse sequence was used to generate T1-weighted images. The imaging parameters were TR/TE 21/ $8 \mathrm{~ms}$, flip angle $30^{\circ}, 240 \mathrm{~mm}$ field of view, $256 \times 192$ in-plane acquisition matrix (interpolated on the scanner to $256 \times 256$ ), and 128 axial slices ( $1.2 \mathrm{~mm}$ thick) covering the whole brain. Then the midsagittal cross sections of the white matter segmented images showing the corpus callosum were extracted and smoothed with 15-pixel-wide FWHM 2D Gaussian kernel (Fig. 1).

\section{Results}

First we fit the white matter density change over age via linear growth model (Eq. (6)). The white matter increase of $2.5 \%$ per year in the genu of the autistic group is statistically significant (uncorrected $P$ value $<0.0014$; corrected $P$ value $<0.16$ ). Other regions of the corpus callosum do not show much age effect. The decrease of 2.5 per year in the midbody of the control group is not statistically significant (uncorrected $P$ value 0.1 ; corrected $P$ value $\approx 1$ ) (Figs. 4 and 5). Since there is no age effect in the splenium, the white matter difference based on the two sample $t$ test in that region should be largely due to the group difference while the white matter difference detected in the genu may be in fact due to a possible age effect. So we refitted GLMs as described in the previous section and computed $F$ statistic maps. We found statistically significant white matter deficiency in the splenium after controlling for age (corrected $P$ value, 0.099; uncorrected $P$ value, $0.005 ; F$ value, 9.47). See Fig. 7 a for the resulting $F$ map. It is interesting to note that the splenium is the region of high anatomical variability for the autistic group (Fig. 2 ). In the splenium, we found nine times more variable white matter density ( $P$ value of 0.0004 ). Comparing GLM results (Fig. 7) with the two sample $t$ test results not taking account for the age effect (Fig. 8), we see similar pattern of statistical map in both cases indicating the age effect in our study is negligible due to the compatible age range between groups.
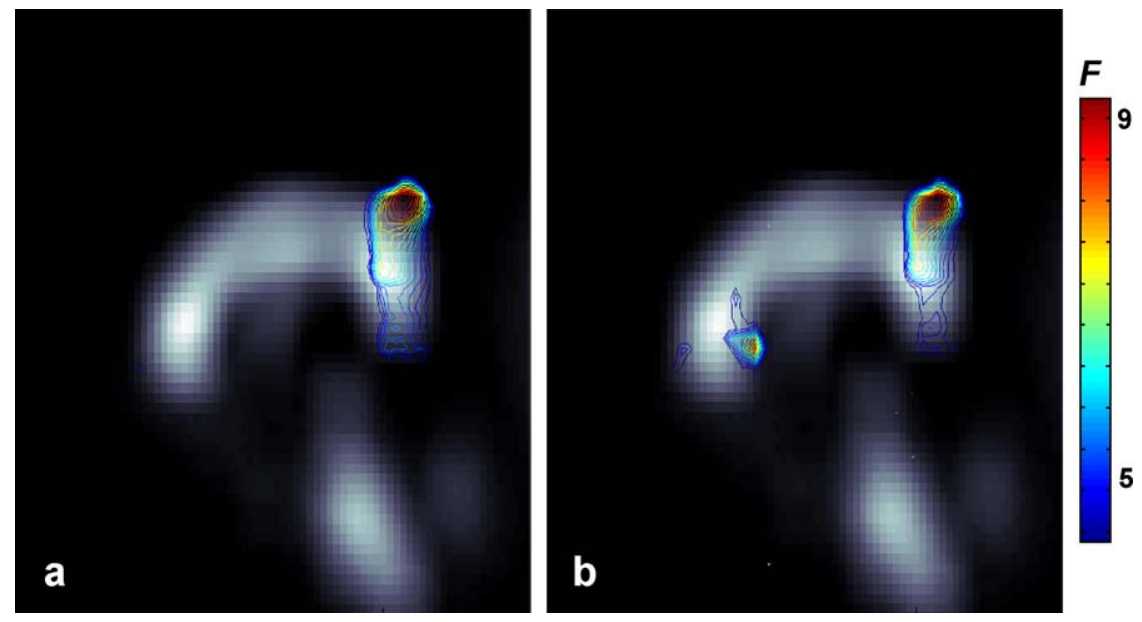

Fig. 7. Contour plots of $F$ map showing the statistically significant white matter density difference. $F$ maps are superimpose on top of the sample mean of the white matter map of 12 control subjects. (a) After controlling for age only. (b) After controlling for both age and total corpus callosum area. It shows less white matter concentration in the splenium for the autistic subjects. 


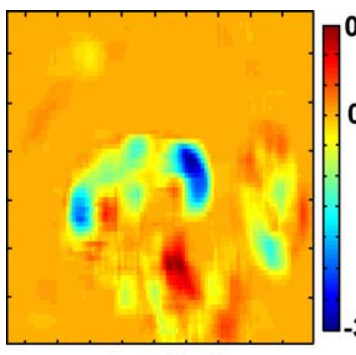

t-statistic

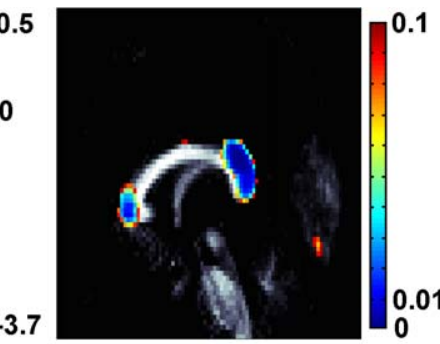

$P$-value

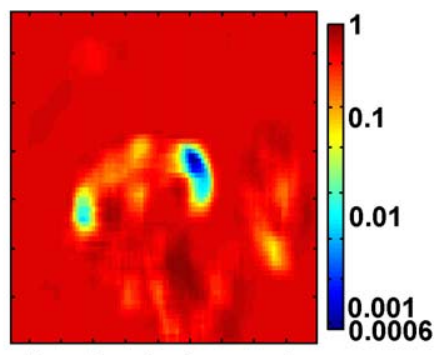

$P$-value in logscale

Fig. 8. Left: two sample $t$ statistics. Middle: $P$ value map thresholded at 0.1 . Right: $P$ value in logarithmic scale. The log scale is useful in displaying small $P$ values. The splenium and the genu of the corpus callosum show huge white matter deficiency in autism compared to control.

Further to account for the possible global corpus callosum size difference among subjects, we have fitted GLM (Eq. (8)) controlling for both age and the total corpus callosum area (Table 1). Due to a somewhat coarse image registration of SPM'99 that is required in VBM (Good et al., 2001a,b), Table 1 shows slight variations in the area estimates although they are not statistically significant. We fitted

area $=\lambda_{1}+\lambda_{2} \cdot$ age $+\beta_{1} \cdot$ group

and tested if area is significantly different between groups, that is, $H_{0}: \beta_{1}=0$. There is no statistically significant total area difference between the groups after image registration ( $P$ value, $0.67 ; F$ value, $1.005)$. It reconfirms the fact that the image normalization has an effect of controlling the total corpus callosum area differences. After fitting (Eq. (8)), we detected the statistically significant less white matter concentration in the splenium (corrected $P$ value, 0.097; uncorrected $P$ value, $0.0049 ; F$ value, 9.62). On the other hand, we also detected less white matter concentration in the genu and rostrum regions (corrected $P$ value, 0.16 ; uncorrected $P$ value, $0.0086 ; F$ value, 8.18 ); however, the result may not pass very conservative $\alpha=0.1$ level test (see Fig. 7b). Note that the ROI-based morphometry using the total corpus callosum area was not able to detect the group difference while the VBM was able to pinpoint the splenium and possibly the genu within the ROI as the regions of white matter concentration difference. This further demonstrates the advantage of using the VBM over the ROI-based morphometry.

To check for possible image processing artifacts, we applied our statistical tests on null data constructed from randomly selecting 8 from 16 autism and 6 from 12 controls and combining them together to form a new group consisting of 14 subjects. Combining the remaining 14 subjects formed the second new group. Afterward, the same image processing and VBM were performed on the null data set revealing no significant result. Note that we are not trying to compute corrected $P$ value via random permutation test but rather to check if our image processing and data analysis procedures might give false positives. A similar approach of generating null data and checking possible image processing artifacts has been proposed in Chung et al. (2003) for a child brain development study.

\section{Discussion}

The $2 \mathrm{D}$ version of the voxel-based morphometry was used in the midsagittal cross section of MRI quantifying the white matter deficiency in high functioning autism. Accounting for an age effect, statistically significant white matter deficiency in the genu, rostrum, and splenium of the corpus callosum was detected in the autistic group, but there is no significant difference in the midbody. This may suggest impaired interhemispheric connectivity in frontal and particularly temporal and occipital regions. It is interesting to note that Piven et al. (1996) found increased volume of the parietal, temporal, and posterior lobes, but not the frontal lobes in autism compared to normal control. Carper et al. (2002) did not find statistically significant frontal, temporal, parietal, and occipital white matter volume differences between the groups in age range 7.5-11.5 years; however, their regression analysis showed that the predicted white matter volume at age 12 is substantially lower in the autistic group. The orbital frontal cortex projects through the rostrum while occipital and inferior temporal cortex project through the splenium (Hardan et al., 2000). Therefore, our findings indirectly suggest the impaired interhemispheric connectivity in those cortical regions.

The deficit in splenium white matter may be associated with the abnormalities in face processing and particularly in the identification of emotion in faces (Dalton et al., 2003). In normal subjects, faces activate the right fusiform area and the verbal identification of the emotion in a face likely requires transfer of information between the hemispheres in the splenium region. The deficit found here in the autism group may at least in part underlie the abnormalities in emotional face processing observed in this group (Dalton et al., 2003).

Linear growth models were fitted for autism and control groups separately to show different pattern of white matter density change over time. Subjects with autism shows lower white matter concentration at the lower age range in almost all parts of the corpus callosum, but the white matter density increases more rapidly over age to catch up with that of the control subjects. A similar result is reported in Courchesne et al. (2001) that shows more cerebral $(10 \%)$ and cerebellar $(39 \%)$ white matter in 2- and 3-year-old autistic subjects but did not find such enlarged white matter volume in older children and adolescents. On the other hand, the normal control group seems to show a higher concentration of the white matter at younger ages but decreasing density in the midbody with increase in age. However, this white matter decrease detected in the midbody is not statistically significant.

The smaller callosal size in the genu and splenium might be attributed to hypoplasia rather than atrophy. In particular, we found a statistically significant $2.5 \%$ year increase of the white matter in the genu for the autistic group.

A similar result was obtained using tensor-based morphometry where Vidal et al. (2003) compared 15 autistic subjects of age $9.9 \pm$ 3.2 years to a group of 13 control subjects of age $10 \pm 2.1$ years. 
They found the most significant reduction of the corpus callosum size in the genu, splenium, and midbody in decreasing order. The slight difference with our voxel-based morphometry result might be due to the different morphometric techniques plus manual segmentation used in Vidal et al. (2003), while no manual segmentation of any sort was applied in our VBM approach.

\section{Acknowledgments}

Authors wish to thank Terry Oacks and Andrew Fox of the Keck Brain Imaging Laboratory, University of Wisconsin, Madison, for valuable discussions on VBM, Kam Tsui of the Department of the Statistics, University of Wisconsin, Madison, for the discussion on the generalized $P$ value, and John Ashburner for pointing out the image orientation convention in SPM'99 package. KeithWorsley of McGill University and Jonathan Taylor of Stanford University provided valuable comments on the Minkowski functionals. This work was funded by Wisconsin Alumni Research Foundation (WARF), NIMH U54 MH066398, and by a NARSAD Distinguished Investigator Award to RJD.

Authors also thank anonymous reviewers for the improvement of the paper.

\section{References}

Abell, F., Krams, M., Ashburner, J., Passingham, R., Friston, K., Frackowiak, R., Happé, F., Frith, C., Frith, U., 1999. The neuroanatomy of autism: a voxel-based whole brain analysis of structural scans. NeuroReport 10, 1647-1651.

Ashburner, J., Friston, K.J., 1997. Multimodal image coregistration and partitioning-A unified framework. NeuroImage 6, 209-217.

Ashburner, J., Friston, K.J., 2000. Voxel-based morphometry-The method. NeuroImage 11, 805-821.

Ashburner, J., Friston, K.J., 2001. Why voxel-based morphometry should be used. NeuroImage 14, 1238-1243.

Benjamini, Y., Hochberg, Y., 1995. Controlling the false discovery rate: a practical and powerful approach to multiple testing. J. R. Stat. Soc, Ser. B 57, 289-300.

Bookstein, F.L., 2001. Voxel-based morphometry should not be used with imperfectly registered images. NeuroImage 14, 1454-1462.

Brambilla, P., Hardan, A., di Nemi, S.U., Perez, J., Soares, J.C., Barale, F., 2003a. Brain anatomy and development in autism: review of structural MRI studies. Brain Res. Bull. 61, 557-569.

Brambilla, P., Nicoletti, M.A., Sassi, R.B., Mallinger, A.G., Frank, E., Kupfer, D.J., Keshavan, M.S., Soares, J.C., 2003b. Magnetic resonance imaging study of corpus callosum abnormalities in patients with bipolar disorder. Biol. Psychiatry 54, 1294-1297.

Carper, R.A., Moses, P., Tigue, Z.D., Courchesne, E., 2002. Cerebral lobes in autism: early hyperplasia and abnormal age effects. NeuroImage 16, $1038-1051$.

Chung, M.K., Worsley, K.J., Paus, T., Cherif, C., Giedd, J.N., Rapoport, J.L., Evans, A.C., 2001. A unified statistical approach to deformationbased morphometry. NeuroImage 14, 595-606.

Chung, M.K., Worsley, K.J., Robbins, S., Paus, P., Taylor, J., Giedd, J.N., Rapoport, J.L., Evans, A.C., 2003. Deformation-based surface morphometry with an application to gray matter deformation. NeuroImage $18,198-213$.

Chung, M.K., Dalton, K.M., Alexander, A.L., Davidson, R.J., 2004. White matter density of corpus callosum in autism: $2 \mathrm{D}$ voxel-based morphometry. TR. 1089. Department of Statistics, University of WisconsinMadison. http://www.stat.wisc.edu/_mcung/papers/autism_tech.pdf.

Courchesne, E., Karns, C.M., Davis, H.R., Ziccardi, R., Carper, R.A., Tigue, Z.D., Chisum, H.J., Moses, P., Pierce, K., Lord, C., Lincoln,
A.J., Pizzo, S., Schreibman, L., Hass, R.H., Akshoomoff, N.A., Courchesne, R.Y., 2001. Unusual brain growth patterns in early life in patients with autistic disorder: an MRI study. Neurology 57, 245-254.

Dalton, K.M., Anderle, M., Fisher, R., Schaeffer, H., Alexander, A.L., Davidson, R.J., 2003. Brain function in individuals diagnosed with autism during discrimination of emotion. The Proceeding of the 9th Annual Meeting of the Organization for Human Brain Mapping, New York, NY.

Egaas, B., Courchesne, E., Saitoh, O., 1995. Reduced size of the corpus callosum in autism. Arch. Neurol. 52, 794-801.

Genovese, C.R., Lazar, N.A., Nichols, T.E., 2002. Thresholding of statistical maps in functional neuroimaging using the false discovery rate. NeuroImage $15,870-878$.

Good, C.D., Johnsrude, I., Ashburner, J., Henson, R.N.A., Riston, K.J., Frackowiak, R.S.J., 2001a. A voxel-based morphometric study of ageing in 465 normal adult human brains. NeuroImage 14, 21-36.

Good, C.D., Johnsrude, I.S., Ashburner, J., Henson, R.N.A., Friston, K.J., Frackowiak, R.S.J., 2001b. A voxel-based morphometric study of ageing in 465 normal adult human brains. NeuroImage 14, 21-36.

Hardan, A.Y., Minshew, N.J., Keshavan, M.S., 2000. Corpus callosum size in autism. Neurology 55, 1033-1036.

Hoffmann, T.J., Chung, M.K., Dalton, K.M., Alexander, A.L., Wahba, G., Davidson, R.J. 2004. Subpixel curvature estimation of the corpus callosum via splines and its application to autism, 10th Annual Meeting of the Organization for Human Brain Mapping. http://www.stat.wisc.edu/ _mchung/papers/HBM2004/HBM2004thomas.html.

Luders, E., Rex, D.E., Narr, K.L., Woods, R.P., Janke, L., Thompson, P.M., Mazziotta, J.C., Toga, A.W., 2003. Relationships between sulcal asymmetries and corpus callosum size: gender and handedness effects. Cereb. Cortex 13, 1084-1093.

Manes, F., Piven, J., Vrancic, V., Plebst, C., Starkstein, S.E., 1999. An MRI study of the corpus callosum and cerebellum in mentally retarded autistic individuals. J. Neuropsychiatry Clin. Neurosci. 11, 470-474.

Mehta, S., Grabowski, T.J., Trivedi, Y., Damasio, H., 2003. Evaluation of voxel-based morphometry for focal lesion detection in individuals. NeuroImage 20, $1438-1454$.

Nichols, T.E., Holmes, A.P., 2002. Nonparametric permutation tests for functional neuroimaging experiments: a primer with examples. Hum. Brain Mapp. 15, 1-25.

Paus, T., Zijdenbos, A., Worsley, K., Collins, D., Blumenthal, L., Giedd, J.N., Rapoport, J.L., Evans, A.C., 1999. Structural maturation of neural pathways in children and adolescents: in vivo study. Science 283, $1908-1911$.

Pettey, D.J., Gee, J.C., 2002. Sexual dimorphism in the corpus callosum: a characterization of local size variations and a classification driven approach to morphometry. NeuroImage 17 1504-1511.

Piven, J., Bailey, A.S., Andreasen, N., 1996. Regional brain enlargement in autism: a magnetic resonance imaging study. J. Am. Acad. Child Adolesc. Psych. 35, 530-536.

Piven, J., Bailey, J., Randon, B.J., Arndt, S., 1997. An MRI study of the corpus callosum in autism. Am. J. Psychiatry 154, 1051-1056.

Salmond, C.H., Ashburner, J., Vargha-Khadem, F., Connelly, A., Gadian, D.G., Friston, K.J., 2002. Distributional assumptions in voxel-based morphometry. NeuroImage 17, 1027-1030.

Shen, D., Davatzikos, C., 2003. Very high-resolution morphometry using mass-preserving deformations and HAMMER elastic registration. NeuroImage $18,28-41$.

Talairach, J., Tournoux, P., 1988. Co-Planar Stereotaxic Atlas of the Human Brain: 3-Dimensional Proportional System - An Approach to Cerebral Imaging. Thieme Medical, New York, NY.

Thompson, P.M., Giedd, J.N., Woods, R.P., MacDonald, D., Evans, A.C., Toga, A.W., 2000. Growth patterns in the developing human brain detected using continuum-mechanical tensor mapping. Nature 404, $190-193$.

Tsui, K.-W., Weerahandi, S., 1989. Generalized $p$-values in significance testing of hypotheses in the presence of nuisance parameter. J. Am. Stat. Assoc. 84, 602-607.

Vidal, C.N., DeVito, T.J., Hayashi, K.M., Drost, D.J., Williamson, P.C., 
Craven-Thuss, B., Herman, D., Sui, Y., Toga, A.W., Nicolson, R., Thompson, P.M., 2003. Detection and visualization of corpus callosum deficits in autistic children using novel anatomical mapping algorithms, Proc. International Society for Magnetic Resonance in Medicine. http:// www.loni.ucla.edu/_thompson/ISMRM2003/cvISMRM2003.html.

Witelson, S.F., 1985. The brain connection: the corpus callosum is larger in left-handers. Science 229, 665-668.

Witelson, S.F., 1989. Hand and sex differences in the isthmus and genu of the human corpus callosum. Brain 112, 799-835.

Worsley, K.J., 1994. Local maxima and the expected Euler characteristic of excursion sets of $\chi^{2}, F$ and $t$ fields. Adv. Appl. Probab. 26, 13-42.

Worsley, K.J., Marrett, S., Neelin, P., Evans, A.C., 1995. A unified statis- tical approach for determining significant signals in location and scale space images of cerebral activation. In: Myers, R., Cunningham, V.J., Bailey, D.L., Jones, T. (Eds.), Quantification of Brain Function Using PET. Academic Press, San Diego, pp. 327-333.

Worsley, K.J., Marrett, S., Neelin, P., Vandal, A.C., Friston, K.J., Evans, A.C., 1996. A unified statistical approach for determining significant signals in images of cerebral activation. Hum. Brain Mapp. 4, $58-73$

Wright, I.C., McGuire, P.K., Poline, J.-B., Travere, J.M., Murray, R.M., Frith, C.D., Frackowiak, R.S.J., Friston, K.J., 1995. A voxel-based method for the statistical analysis of gray and white matter density applied to schizophrenia. NeuroImage 2, 244-252. 